\title{
Multilingual Address and Counter-Orientalist Practice
}

English version

\section{Thomas Lamarre}

\section{(2) OpenEdition Journals}

Electronic version

URL: http://journals.openedition.org/transtexts/181

DOI: 10.4000/transtexts.181

ISSN: 2105-2549

\section{Publisher}

Gregory B. Lee

Printed version

Date of publication: 1 May 2006

Number of pages: $56-57$

ISSN: 1771-2084

\section{Electronic reference}

Thomas Lamarre, «Multilingual Address and Counter-Orientalist Practice», Transtext(e)s Transcultures 跨文本跨文化 [Online], 1 | 2006, Online since 13 September 2009, connection on 05 May 2019. URL: http://journals.openedition.org/transtexts/181; DOI : 10.4000/transtexts.181 


\title{
Multilingual address and counter-orientalist practice BY Thomas Lamarre
}

\begin{abstract}
In 2003, in a new afterword to Orientalism entitled "Orientalism 25 Years Later," Edward Said noted that the general understanding of the Middle East, the Arabs and Islam had not really improved in the intervening years. In the aftermath of the events of September 11, 2001 and Britain and the United States's illegal invasion and imperial occupation of Afghanistan and Iraq, representations of the Middle East bolstered the notion of an implacable clash of civilizations. Impossibly, ironically, Orientalism remained alive and well. And all the questions initially posed around Said's work returned with new urgence. What exactly is the relation between representation and imperialism ? Does imperialism rely so heavily on historically accumulated misrepresentations of its Others that imperialism cannot function without them ? Will well-executed attacks on systematic mispresentations of the "Orient" - or that other quasi-fantastical discursive construct "Asia" — help put an end to empire ?

Although twenty-five years after Orientalism Said endorsed "worldly humanism" in opposition to imperialism (subtitling his afterword "Worldly Humanism versus the Empire-builders"), the original book offered a broader range of ways of thinking about Orientalism. Indeed some would say that it offered too many different approaches. For many commentators have stated, the book remains unable to decide whether Orientalism is (a) a systematic misrepresentation of a region, (b) an innately human process of ethnocentric "othering" (establishing a sense of self by inventing and categorically debasing an other), or (c) a historically specific formation of knowledge, that is, a form of disciplinary power. While acknowledging the moral force behind Said's arguments, Aijaz Ahmad, for instance, wrote lucidly of how Said's many takes on Orientalism resulted in a mish-mash of incompatible stances. It was as if, in his outrage over the continued abjection of the Arabs, Islam and the Middle East in the Western academy, Said had thrown the net so wide that almost any representation of any Other to the West at any historical moment appeared to constitute Orientalism.

The vastness of Orientalism's applications, its fluidity and capaciousness, continues to trouble some commentators. Some have argued that all the fuss over Orientalism is merely a way of pointing at a problem - that of power and representation - without providing any real insights into how power or representation operates (beyond a flattened self/other dialectic). Yet is the protean quality of Said's analysis of Orientalism truly such a liability right now ? In North America at least Said's book has had an especially important and salutary effect on domains of the Academy, especially in so-called Area Studies. His questions about representation and power forced scholars to question academic strategies of representation and to think in terms of knowledge/power formations for the first time. This came as a revelation to many who had entered the field simply to generate positivistic data related to the study of a foreign
\end{abstract}


country without any thoughts about the effects of the organization or disciplinary construction of knowledge.

The vast critical net of Orientalism is, in effect, one of the points of departure for Transtext(e)s Transcultures, and for the exploration in inaugural issue of the problem of power and representation from a number of different angles, always with an eye to "Western" representations of others - or, more precisely, with an eye to how a modern history or a modern subject opens to alterity. What do we make of this repetition of the Orientalist problem and Orientalist effects in different contexts? Are we dealing with ethnocentrism in general, subjectivity in general, representation in general ? Is there something specific to Western ethnocentrism that makes it into Orientalism ? If so, when and how does it appear, and how does it transform ?

Such questions emerged in the wake of Orientalism, as scholars of Japan, Korea and China, for instance, recognized many of the same 'Orientalizing' operations at work in academic constructions of their Orient, that is, the region geographically known as Northeast Asia. While countries in that region had known Western imperialism and had been subject to analogous formations of "orientalizing" knowledge, the effects of Orientalism had been different. Or were there different Orientalisms? Or was it simply that a country such as Japan produced a different response to Western imperialism, developing in turn its own "orientalizing" stance toward its imperial colonies in East Asia and the South Pacific ? Here the problem of Orientalism meshes with that of Western modernity, a process of rupture and reinscription. Here, too, the problem of power and representation intersects that of language and address. After all, what is the Orientalist effect but a mode of address that constructs a sense of being at home in language ? As Said's long chapters on the legacy of Orientalist philology attest, Orientalism assumes that homogenous languages preexist their actual usage or figuration, that those speakers were, are, and will remain at home in them - by force, if necessary.

In Translation and Subjectivity, Naoki Sakai writes of a "heterolingual" mode of address. Heterolingual address - we might also call it "multilingual" address — is not a matter of engaging different audiences speaking or reading different national languages, say, English, Chinese, and French. It does not presuppose the preexistence of homogeneous national languages. Rather it addresses the discontinuity, difference, or alterity that comes into play within any language; it engages the multiplicity within language not the plurality of languages. Usually, the act of translation wipes away heterolingual address by creating the sense of two homogenous languages and a movement from one to the other rather than a movement within language itself.

The challenge of Transtext(e)s Transcultures lies in its potential to disrupt the homolingual mode of address, thus opening the possibility of a counter-Orientalist practice. Of course, the presentation of texts in Chinese, French, and English will not in itself trouble anyone - not until the co-presence of those languages begins to disturb the homolingual mode of address. (While global English today guarantees the homolingual propriety and purity of national languages, and indeed the aspiration to whiteness implicit therein, one cannot say that French and Chinese do not operate in this way as well.) Which is to say, the challenge of this journal lies not in a simple plurality of languages, in a simple gesture of representation of differing language homes. Rather it is as readers and contributors start to read and write with a sense of multiple orientation in "their" language of address that the co-presence of different national languages will commence its work of disorientation, countering the Orientalist practices entwined with the imperialism that today would strangle the globe in its homolingual embrace. 


\section{Adresse plurilingue et pratique contre-orientaliste}

En 2003, dans une nouvelle postface à Orientalism intitulée «L'Orientalisme, 25 ans plus tard », Edward Said relevait que la compréhension générale du Moyen-Orient, des Arabes et de I'Islam ne s'était pas vraiment améliorée depuis la publication de son livre. A la suite des évènements du 11 septembre 2001, de l'invasion illégale et de l'occupation impérialiste par les Etats-Unis et la Grande-Bretagne de l'Afghanistan et de l'Irak, les représentations du Moyen-Orient ont renforcé l'idée d'un implacable choc des civilisations. Contre toute attente, ironiquement, l'Orientalisme a survécu et se porte très bien. Et toutes les questions que le travail de Said avait soulevées à l'origine resurgissent et sont de nouveau urgentes. Quelle est précisément la relation entre représentation et impérialisme ? Les fondements de l'impérialisme sont-ils si profondément ancrés dans des représentations erronées de ses Autres, accumulées au fil du temps, qu'il ne peut fonctionner sans elles ? Une critique efficace de ces représentations systématiques de l' "Orient »- ou de l' "Asie », cette autre construction discursive presque fantastique - permettra-t-elle de mettre fin à l'empire ?

Vingt-cinq ans après Orientalism, Said en appelait à un « humanisme mondial » en opposition à l'impérialisme (donnant à sa postface le sous-titre : "L'humanisme mondial contre les faiseurs d'Empire »). L'ouvrage proposait cependant à l'origine un plus grand nombre de possibilités de penser l'Orientalisme. Certains dirent en fait que trop d'approches différentes étaient proposées car, comme l'ont affirmé de nombreux analystes, cet ouvrage ne parvient pas à dire si l'Orientalisme est (a) la représentation erronée systématique d'une région, (b) un phénomène naturel qui pousse les hommes à « rendre-autre » de manière ethnocentrique (à établir une conscience de soi à travers l'invention et la dépréciation systématique d'un autre), ou (c) une formation du savoir historiquement datée, c'est à dire, une forme de pouvoir disciplinaire. Tout en reconnaissant la force morale des arguments de Said, Aijaz Ahmad, par exemple, a démontré avec lucidité dans ses écrits comment les nombreuses attaques de Said contre l'Orientalisme ont produit un mélange confus de postures incompatibles. C'était comme si, emporté par son indignation vis à vis du mépris continu des Arabes, de l'Islam et du Moyen-Orient dans les académies occidentales, Said avait lancé son filet si loin que presque toute représentation d'un Autre de l'Occident, quel que soit le moment historique, semblait être constitutive de l'Orientalisme.

L'étendue des applications de l'Orientalisme, sa fluidité et l'infini de son contenu continuent de troubler certains analystes. Certains ont affirmé que tout le tapage autour de l'Orientalisme était simplement un moyen de mettre l'accent sur un problème - celui du pouvoir et de la représentation - sans apporter un éclairage véritable sur la manière dont le pouvoir ou la représentation fonctionnent (au-delà d'une dialectique soi/autre aplanie). Et pourtant l'aspect variable des analyses de l'Orientalisme par Said est-il vraiment un tel handicap à I'heure actuelle ? En Amérique du Nord au moins, le livre de Said a eu un effet particulièrement important et salutaire au sein du monde académique, spécifiquement dans le champ des dites Aires Culturelles. Ses questions sur la représentation et le pouvoir ont obligé les chercheurs à 
mettre en cause les stratégies de représentation au sein du monde académique et à penser en termes de formations savoir/pouvoir pour la première fois. Ce fut une révélation pour tous ceux qui avaient intégré le champ des Aires Culturelles avec pour unique objectif de générer des données scientifiques positivistes en lien avec l'étude de pays étrangers sans penser aux effets produits par cette organisation du savoir et sa construction disciplinaire.

Le vaste espace critique de l'Orientalisme est, en effet, l'un des points de départ de Transtext(e)s Transcultures, et de l'exploration, dans ce premier numéro, du pouvoir et de la représentation à partir de différentes perspectives, en gardant toujours un l'œil sur les représentations « occidentales » des autres - ou, plus précisément, sur comment l'histoire moderne ou un sujet moderne s'ouvre à l'altérité. Que faisons-nous de la répétition de la question Orientaliste et des effets de l'Orientalisme dans différents contextes ? S'agit-il d'ethnocentrisme en général, de la subjectivité en général, de la représentation en général ? L'ethnocentrisme occidental possède-t-il une caractéristique spécifique qui entraîne l'Orientalisme ? Si c'est le cas, quand et comment cela se produit-il, et comment cela évolue-t-il ? De telles questions ont fait surface dans le sillage de Orientalism, lorsque des chercheurs du Japon, de Corée ou de Chine, par exemple, se sont rendu compte qu'un même processus d'« orientalisation » était à l'œuvre sous de nombreuses formes dans les constructions académiques de leur Orient, c'est à dire l'espace géographique connu sous le nom d'Asie du Nord-est. Bien que les pays cette région aient connu l'impérialisme Occidental et aient été soumis à des formes analogues de savoir « orientalisant », les effets de l'Orientalisme ont été différents selon les pays. S'agissait-il en fait d' Orientalismes différents ? Ou bien simplement du fait qu'un pays tel que le Japon avait répondu de manière différente à l'impérialisme Occidental et développé à son tour son propre positionnement « orientalisant » envers ses colonies impériales de l'Asie de l'Est et du Pacifique Sud. Le problème de l'Orientalisme se mêle ici à celui de la modernité occidentale dans un processus de rupture et de réinscription. De même, la question du pouvoir et de la représentation croise celle du langage et de l'adresse. Finalement, qu'est-ce que l'effet orientaliste, sinon un mode d'adresse qui crée l'impression de se sentir chez soi dans la langue ? Comme l'atteste les longs chapitres de Said sur le legs de la philologie Orientaliste, dans le domaine de la philologie, l'Orientalisme considère que des langues homogènes préexistent à leur usages ou formes présentes, et que leurs locuteurs étaient chez eux dans ces langues, le sont encore et le resteront, de gré ou de force.

Dans Translation and Subjectivity, Naoki Sakai mentionne un mode d'adresse hétérolingue. Ce mode d'adresse - que nous pourrions appeler adresse «multilingue » n'a rien à voir avec l'idée de relier différents publics parlant ou lisant dans différentes langues nationales, comme l'anglais, le français ou le chinois. Cela ne présuppose pas la préexistence de langues nationales homogènes, mais implique plutôt la discontinuité, la différence, ou l'altérité qui entre en jeu quelle que soit la langue, et engage la multiplicité à l'intérieur de la langue et non la pluralité des langues. En général, l'acte de traduction évacue l'adresse hétérolingue en créant l'impression de deux langues homogènes et l'idée d'un mouvement de l'une à l'autre plutôt qu'un mouvement à l'intérieur de la langue elle-même.

Le défi à relever pour Transtext(e)s Transcultures consiste en sa capacité à bousculer le mode d'adresse homolingue, offrant ainsi la possibilité d'une pratique contre-orientaliste. A l'évidence, personne ne sera troublé par la présentation de textes en chinois, français et anglais - du moins jusqu'à ce que la co-présence de ces langues commence à perturber 
le mode d'adresse homolingue. (Si l'anglais mondialisé est aujourd'hui le garant de la dimension homolingue et de la pureté des langues nationales, et ainsi de l'aspiration à la blanchitude que cela sous-entend, on ne peut pas dire que le français et le chinois n'opèrent pas eux aussi de cette façon.) Ce qui signifie que le défi qui attend ce journal n'est pas simplement d'accueillir une pluralité de langages et ne se situe pas non plus dans un simple geste de représentation de différents foyers de langages. C'est plutôt lorsque les lecteurs et contributeurs commencent à lire et écrire avec l'idée d'une orientation multiple dans «leur » langue d'adresse que la co-présence de différentes langues nationales commence son travaille de désorientation, contestant ainsi les pratiques orientalistes imbriquées dans l'impérialisme qui étrangle aujourd'hui le globe de son étreinte homolingue.

2003年，爱德华. 萨伊德（Edward Said）在其为《东方主义》所撰名为《东方主 义: 25年之后》的跋中指出, 在这段时期内，对中东、阿拉伯人、伊斯兰教的 总体认识并没有实质性的改善。在 9.11 事件以及美英对阿富汗和伊拉克的非法 侵犯和帝国主义式的侵占中, 有关中东的诸多“表象”进一步突显了一种不可调 和的文明冲突的观念。看似荒谬, 然而不无讽刺意味的是, 东方主义不仅依然 存在, 而且还很根深蒂固。并且, 萨伊德书中率先提出的所有问题仍具有紧迫 性。表征与帝国主义间的关系到底为何? 帝国主义脱离了历史积累下来的有 关其“他者”的错误表征是不能够运行的, 它是否如此依赖于此? 针对有关“东 方”的系统性错误表征而展开的强烈攻击，或者其它对“亚洲”的半幻想式的话 语建构, 能否有助于结束帝国主义?

虽然在完成《东方主义》25年后，萨伊德声明支持与帝国主义相对的“世界性 人道主义”（其所作跋的副题为《世界性人道主义与帝国建构者们》），但是 其原作为对东方主义进行反思提供了更广阔的视野。实际上, 有人可能会说该 书提供了太多不同的手段。因为, 有许多评论员曾经指出, 这本书最终仍然未 能表明东方主义到底是（a）对一个地区的系统性的错误表征，还是（b）一种 人类内在的族群中心主义的“他者化”过程（通过建构和分门别类地贬低某个他 者来建立一种自我感), 又或者是 (c) 一种历史上特殊的知识形成过程, 也 就是说, 一种规戒权力的形式。比如说, 阿加兹·阿哈默德（Aijaz Ahmad）等人 在认同萨伊德观点背后道德力量的同时, 清楚地指出萨伊德对东方主义的许多 尝试，所炮制出来的是一锅各种立场自相矛盾的大杂烩。好像是在其对于长期 以来, 西方学术界鄙弃阿拉伯人、伊斯兰教和中东的愤怒中, 萨伊德的网大得 以及至于，几乎所有有关对于西方而言的任何“他者”的任何表征，在任何历史 时刻看起来都是在建构着“东方主义”。

《东方主义》应用之广泛, 及其流动性和包容性一直使某些批评者感到困惑。 有些评论员指出, 有关于东方主义的所有争议, 仅仅是一种指出一个问题的方 式一一权力与表征问题, 而没有提供有关于权力与表征（在一种被征服的自 我/他者的辩证逻辑之外) 如何运作的任何真正的洞察力。而如今, 萨伊德对 东方主义分析之变化多端的特性是否真是这样一种缺陷呢? 至少在北美地区, 
萨伊德的书已对诸多学术领域产生过异常重要而有益的影响, 尤其是对所谓 的“区域研究”而言。他有关表征与权力的问题迫使学者们置疑有关表征的学术 策略，并第一次以知识/权力构成的话语形式来思考问题。这一点对于很多人 来说是一种启示, 他们进入到这一研究领域, 仅是为了制造一些与某个外国相 关的实证性资料, 而并没有考虑到知识的组织或者学科知识架构的影响。

实际上, 建立广泛的有关东方主义的批评网络，是《跨文本跨文化》的出发 点之一，也是首刊藉此开始从众多不同角度来探讨权力和表征问题的出发点之 一，本刊始终关注“西方”对于他者的表征问题，或者更确切地讲，是一直关注 现代历史或者现代主体如何对于他性保持开放的问题。我们该如何审视东方主 义问题以及东方主义影响在不同语境中的重复? 我们面对的是不是一般意义上 的族群中心主义、主体性和表征? 西方的族群中心主义是否具有某些特殊性以 使其发展成为东方主义的? 如果答案是肯定的，那么这些特殊成份在什么时候 以什么形式呈现? 而它们又是如何转化的呢?

《东方主义》出现以后, 一些问题则随之涌现出来, 例如日本、韩国和中国等 国家的学者们认识到，在其有关“东方”（即地理上被称之为东北亚的地区）的 学术建构中，存在着许多同样的“东方化”运作。尽管这一地区的诸多国家早已 对西方的帝国主义有所了解，并且早就曾遭受过有关“东方化”知识的类似的形 成过程的影响, 但是东方主义在这些国家的影响有所不同。或者究竟存在不同 的东方主义与否? 或者是否仅仅是一个像日本这样的国家对西方帝国主义进行 了不同的回应，以其在东亚和南太洋地区的帝国主义殖民地为目标，先后展现 了其自身的“东方化”姿态? 在此，东方主义问题实际上跟西方现代性问题相吻 合，而后者是一个断裂与重新刻划的过程。在这里，权力和表征的问题同样与 语言及表述的问题相交叉。除了作为建立一种精通语言的感觉的表述模式外, 东方主义的影响终究是什么呢? 正如萨伊德在关于东方主义文献学遗产的长篇 章节中所证明的，东方主义认为诸多同质性的语言先于其现行用法或外在形式 而存在, 那些语言实践者过去会, 现在会, 将来也会仍旧精通于之一一如果需 要的话, 则可能会强迫地进行。

酒井直树（Naoki Sakai）在《翻译与主体性》中写到一种“异质语言”表述模式。 异质语言表述，我们或许也可以称之为“多语”表述，所涉及的并不是让不同的 受众讲不同民族国家的语言，或用之阅读，如英语、汉语和法语。这并不预示 着同质性民族国家语言的先在。相反，它指的是任何一种语言内部存在的不连 续性、差异或他性; 它讲的是语言内部的多样性而非诸多语言的多元性。一般 来说, 翻译实践消除异质语言的表述, 是通过创造两种同质性语言的感觉, 以 及从一种语言到另一种语言的运转, 而非在语言自身内部运转的感觉而实现。 《跨文本 跨文化》的挑战在于其瓦解这种同质语言表述模式的潜能, 这样以 来就开创了一种“反东方主义实践”的可能性。当然，汉语、法语和英语文本的 呈现本身并不会困扰任何人，只有那些语言的共同呈现才足以打破这种同质语 言的表述模式。（尽管如今全球性的英语确保了民族国家语言的同质语言特性 和纯洁性，而且实际上向白人世界靠近的那种渴求不言自明，但任何人都不 能说, 法语和汉语就不能以同样的方式运作。）也就是说，这一刊物的挑战不 在于简单的语言之多元性, 而是一种呈现不同语言家族的简单姿态。更确切地 讲，只能当读者与作者开始带着一种在“其自身”语言表述中的多重倾向的感觉 进行阅读和写作的时候，不同民族国家语言的共同呈现才会开始起到迷向的作 用，反击与帝国主义缠绕在一起的东方主义的实践，而前者在当今则有可能会 将我们的世界扼杀在其同质语言的怀抱中。 